\title{
Measurement of the Proton-Air Cross Section with Telescope Array's Middle Drum Detector and Surface Array in Hybrid Mode.
}

\author{
Rasha Abbasi*, John Belz, for the Telescope Array Collaboration ${ }^{\dagger}$ \\ High Energy Astrophysics Institute and Department of Physics and Astronomy, University of \\ Utah, Salt Lake City, Utah, USA \\ E-mail: rasha@cosmic.utah.edu \\ E-mail: belz@cosmic.utah.edu
}

\begin{abstract}
In this work we report on the measurement of the proton-air inelastic cross section $\sigma_{\mathrm{p} \text {-air }}^{\text {ine }}$ using data collected by the Telescope Array (TA) detector. Based on the measurement of $\sigma_{\mathrm{p}-\text { air }}^{\text {inel }}$, the proton-proton cross section $\sigma_{\mathrm{p}-\mathrm{p}}$ is subsequently inferred from the Glauber Formalism and the QCD-inspired fit of Block, Halzen and Stanev. The use of cosmic ray events at ultra high energies enables the measurement of this fundamental quantity at energies currently inaccessible with particle accelerators. The data used in this report was collected over five years using hybrid events from the TA Middle Drum fluorescence detector as well as the TA surface detector array.
\end{abstract}

The 34th International Cosmic Ray Conference,

30 July- 6 August, 2015

The Hague, The Netherlands

\footnotetext{
* Speaker.

${ }^{\dagger}$ For full author list and acknowledgments http://www.telescopearray.org/images/papers/ICRC2015-authorlist.pdf
} 


\section{Introduction}

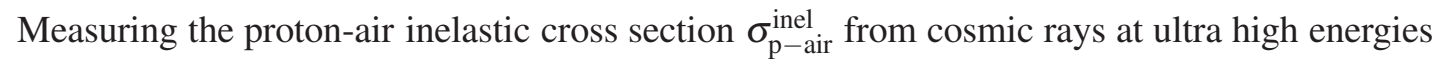
allows us to achieve knowledge on a fundamental particle property that we are unable to attain with measurement at current accelerators. Such a fundamental measurement is important to both constraining the high energy models and to verifying fundamental physics assumptions. The high energy models are in better agreement at lower energies, below $10^{15} \mathrm{eV}$, where they are tuned to measurements on multi-particle production provided by particle accelerators. However, at high energies, above $10^{15} \mathrm{eV}$, models rely solely on theoretical expectations [1].

In this proceeding, we report on the measurement of the proton-air inelastic cross section $\sigma_{\mathrm{p}-\text { air }}^{\text {inel }}$ using the Telescope Array detector (TA). The method used in this calculation is "the $K$ Factor method", where the $K$-Factor method assumes a proportionality between the tail of the the observed shower maximum $X_{\max }$ distribution and the first point of the proton-air interaction slant depth $X_{1}$. Furthermore, the proton-proton cross section $\sigma_{\mathrm{p}-\mathrm{p}}$ is calculated using Glauber theory together with the Block, Halzen, and Stanev (BHS) QCD inspired fit ( [2], [3]). The BHS fit is consistent with unitarity and fits the Fermilab Tevatron Collider data of $\sigma_{\mathrm{p}-\mathrm{p}}[4]$.

\section{Analysis}

In this proceeding we determine the value of $\sigma_{p \text {-air }}^{\text {inel }}$ using the $K$-Factor method. This method infers the attenuation length and hence the cross section value from the exponential tail of the $X_{\max }$ distribution. This is assuming that the tail of the $X_{\max }$ distribution is comprised of the most penetrating/lighter particles (protons). The tail of the $X_{\max }$ distribution is fit to the exponential $\exp \left(\frac{-X_{\max }}{\Lambda_{m}}\right)$, where $\Lambda_{m}$ is the attenuation length. $\Lambda_{m}$ is proportional to the interaction length $\lambda_{p-a i r}$ :

$$
\Lambda_{m}=K \lambda_{\mathrm{p}-\text { air }}=K \frac{14.45 m_{p}}{\sigma_{\mathrm{p}-\text { air }}^{\text {inel }}}
$$

where $K$ is a constant. The departure of $K$ from unity depends on the nucleon and meson cross section with the light nuclear atmospheric target [5].

From the definition of $K$, it is clear that $K$ is model dependent. A study is implemented to investigate the level of the model dependence of the value of $\mathrm{K}$. The high energy models used in this study are QGSJETII.4 [6], QGSJET01 [7], SIBYLL [8], and EPOS-LHC [9]. This study is accomplished using the one-dimensional air shower Monte Carlo program CONEX4.37 ( [10], [11], [12]). It is worth noting that the shower parameters obtained with CONEX are consistent with that obtained with CORISKA [10].

Using CONEX the value of $K$ is determined by simulating 10,000 events between $10^{18.3}$ and $10^{19.3}$ at 0.1 in $\log _{10}(\mathrm{E}(\mathrm{EeV}))$ in energy intervals. The value of $K$ is calculated for each high energy model for each energy bin by obtaining the values of $\Lambda_{m}$ and $\lambda_{\mathrm{p} \text {-air }}$ for that model. The value of $\Lambda_{m}$ and therefore $K$ for each of the data sets is impacted by the choice of the lower edge of the fit range $\left(X_{\text {irange }}\right)$ in the exponential fit to the tail of the $X_{\max }$ distribution.

The choice of $X_{\text {irange }}$ is determined from data, were it was decided at $X_{\text {irange }}=<X_{\max }>$ $+40 \mathrm{~g} / \mathrm{cm}^{2}$. The choice was based on both the stability of the value of $\Lambda_{m}$ at the determined 
$X_{\text {irange}}$, while maximizing the number of events in the tail of the distribution. The same relative shift is later used in the high energy model simulations to determine $X_{\text {irange }}$ and consequently $K$.

It is also important to note that in addition to CONEX we have also used CORSIKA [13] to simulate three-dimensional cosmic ray showers. These showers are propagated through the Fluorescence Detector (FD) and the Surface Detector (SD) part of the TA detector. The successfully detected showers are then reconstructed, after which the pattern recognition cuts were applied [14]. The value of $\Lambda_{m}$ is then determined and is found to be consistent with that obtained with CONEX (pure thrown information) particularly around the selected choice of $X_{\text {irange }}$.

The value of $K$ is calculated for each simulated data set at 0.1 in $\log _{10}(\mathrm{E}(\mathrm{EeV}))$ in energy intervals between the energies of $10^{18.3}$ and $10^{19.3} \mathrm{eV}$. The values of $K$ were found stable around the average value from these data sets. Such stability of $K$ around the average shows that $K$ is independent of energy and justifies the use of a single average value over the range of interest.

To confirm that the value of $K$ obtained is valid to reproduce the interaction length of the model, the value of $\lambda_{\mathrm{p}-\text { air }}$ is reconstructed for each data set and compared to that obtained from the $X_{1}$ distributions for each of the studied high energy models. The result was that the reconstructed values of $\lambda_{\mathrm{p}-\text { air }}$ for the several energy data sets for each of the high energy models was consistent with the model $\lambda_{\mathrm{p} \text {-air }}$ values within the statistical fluctuations. This ensures that the values of $K$ obtained in this study describe the values of $K$ of the studied high energy models correctly.

The $K$-Factor determined in the procedure described above is dependent on the hadronic interaction model used in the air shower Monte Carlo simulation. The resultant values of $K$ determined for these models are summarized in Table 1.

\begin{tabular}{|l|l|}
\hline Model & $K$ \\
\hline QGSJETII.4 & $1.15 \pm 0.01$ \\
\hline QGSJET01 & $1.22 \pm 0.01$ \\
\hline SIBYLL & $1.18 \pm 0.01$ \\
\hline EPOS-LHC & $1.19 \pm 0.01$ \\
\hline
\end{tabular}

Table 1: The value of $K$ obtained for each of the high energy models. Each $K$ listed is the single average value of $K$ over the energy range of $10^{18.3}-10^{19.3}$. Note that the values of $K$ shows a $\sim 3 \%$ model uncertainty.

The $K$ value used in the first UHECR experimental result on the proton-air cross section with the Fly's Eye experiment is 1.6 [15]. It is interesting to note the continuous decrease in the value of $K$ as full Monte Carlo simulations became available. The comparison of the $K$ value arrived at, solely using the continuously developed and updated high energy cosmic ray models, nevertheless diverged, approximately 7\% [16]. With time the high energy models evolving with the LHC data do indeed converge on the value of $K$ with model uncertainty of $3 \%$. This makes the $K$-Value method a weakly model dependent, reliable method to use in calculating the proton-air inelastic cross section $\sigma_{\mathrm{p}-\text { air }}^{\text {inel }}$.

\section{Proton-Air Cross Section:}

The data used in this analysis is the Telescope Array Middle Drum (MD)-Surface Detector (SD) hybrid 439 events collected between May-2008 and May-2013. Figure 1 shows the $X_{\max }$ 


\begin{tabular}{|l|l|}
\hline Model & $\sigma_{\mathrm{p}-\text { air }}^{\text {inel }} \mathrm{mb}$ \\
\hline QGSJETII.4 & $550.3 \pm 68.5$ \\
\hline QGSJET01 & $583.7 \pm 72.6$ \\
\hline SIBYLL & $564.6 \pm 70.2$ \\
\hline EPOS-LHC & $569.4 \pm 70.8$ \\
\hline
\end{tabular}

Table 2: The high energy model vs. the $\sigma_{\mathrm{p}-\text { air }}^{\text {inel }}$ in mb obtained for that high energy model.

distribution together with the exponential unbinned maximum likelihood fit to the tail between 790 and $1000 \mathrm{~g} / \mathrm{cm}^{2}$, the $\Lambda_{m}$ value from the fit is found to be $\left(50.47 \pm 6.26[\right.$ Stat.] $) \mathrm{g} / \mathrm{cm}^{2}$.

Consecutively the value of $\sigma_{\mathrm{p}-\text { air }}^{\text {inel }}$ is determined using Equation 2.1. The values of $\sigma_{\mathrm{p}-\text { air }}^{\text {inel }}$ for all the considered hadronic interaction models are determined and tabulated in Table 2. The final value of the proton-air cross inelastic section reported by the Telescope Array collaboration is the average value of the $\sigma_{\mathrm{p}-\text { air }}^{\text {inel }}$ obtained by the high energy models QGJSETII.4, QGSJET01, SIBYLL, and EPOS-LHC and is found to be equal to $(567.0 \pm 70.5[$ Stat.] $) \mathrm{mb}$.

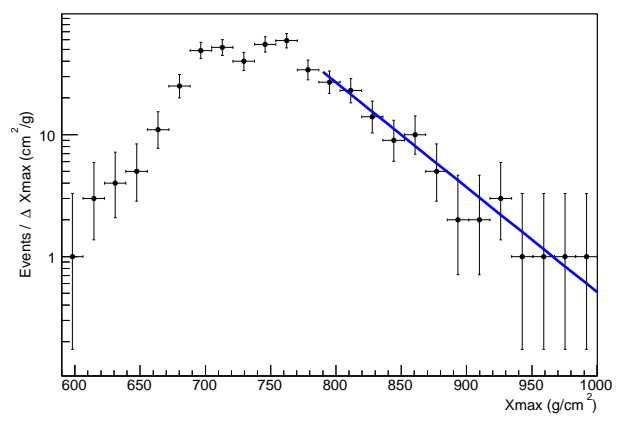

Figure 1: The number of events per $X_{\max }$ bin $\left(\Delta X_{\max }\right)$ vs. $X_{\max }$ in $\mathrm{g} / \mathrm{cm}^{2}$ for the Telescope Array data with the energy between $10^{18.3}$ and $10^{19.3} \mathrm{eV}$. The line is the exponential fit to the slope.

In order to quantify the systematic uncertainties on the proton-air cross section obtained using the $K$-Factor method a few different checks were applied. First, the systematic value from the hadronic interaction model dependence of the $\sigma_{\mathrm{p}-\text { air }}^{\text {inel }}$ value is calculated. In addition, the systematic

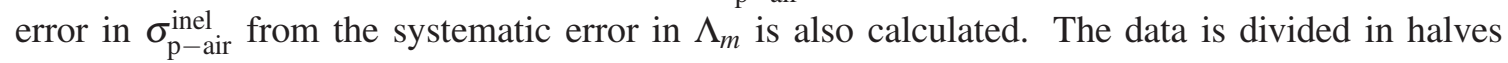
based on the zenith angle of the events, the distance of the shower using the impact parameter, and finally the energy of the events. The attenuation lengths resulting from all these subsets are consistent within the statistical fluctuations.

Moreover, the systematic effect of possible energy dependent bias in the $X_{\max }$ distribution was studied. This is done by shifting the values of $X_{\max }$ by their elongation rate prior to fitting. The value of $\Lambda_{m}$ is calculated and the systematic effect from a possible energy bias was found to be negligible.

The next check is calculating the systematic uncertainty that originates from the detector bias. This includes the bias that occurs from detecting the events, reconstructing the events, and applying the needed cuts to the events. This check is investigated by comparing the result of the attenuation 


\begin{tabular}{|l|l|}
\hline Systematic source & Systematics $(\mathrm{mb})$ \\
\hline Model Dependence & $( \pm 17)$ \\
\hline $10 \%$ Helium & -9 \\
\hline $20 \%$ Helium & -18 \\
\hline $50 \%$ Helium & -42 \\
\hline Gamma & +23 \\
\hline $\begin{array}{l}\text { Total } \\
(20 \% \text { Helium })\end{array}$ & $(+29,-25)$ \\
\hline
\end{tabular}

Table 3: The systematic uncertainties of the proton-air cross section estimated for several sources. The total uncertainty was calculated in quadrature using the error for the $20 \%$ helium case.

length $\Lambda_{m}$ of the simulated shower thrown without any detector effects to the attenuation length obtained from a three-dimensional shower simulation using CORSIKA propagated through the detector and reconstructed successfully including the pattern recognition cuts. The value of $\Lambda_{m}$ was found to be consistent, for all the high energy models, between the thrown events and the reconstructed events with pattern recognition applied. Therefore, the detector bias systematic effect on the $\Lambda_{m}$ value is negligible.

In addition, a fraction of the high energy cosmic rays detected and used in this study are possibly photons. studies placing an upper limit on the integral flux and the fraction of the primary cosmic ray photons for energies greater than $10^{18} \mathrm{eV}$ ( [17], [18]). In this study, the lowest derived limit on the photon fraction is used and is $<1 \%$ [19].

The presence of other elements in the data beside proton is also studied. This includes iron, helium, and CNO. The maximum contribution was found to be from helium (deepest $X_{\max }$ distribution). A contribution of $10 \%, 20 \%$, and $50 \%$ from helium and the systematic error associated with such contribution is reported. Table 3 summarizes the systematic checks for the proton-air cross section, including the final systematic uncertainty.

We summarize the result of the our proton-air cross section obtained using the $K$-Factor method described previously together with the systematic checks obtained to be equal to

$$
\sigma_{\mathrm{p}-\mathrm{air}}^{\text {inel }}=567.0 \pm 70.5[\text { Stat. }]_{-25}^{+29}[\text { Sys. }] \mathrm{mb} .
$$

This is obtained at an average energy of $10^{18.68} \mathrm{eV}$. The result of the proton-air cross section is then compared to the results obtained from various experimental results. In addition, the experimental results of the high energy models (QGSJETII.4, QGSJET01, SIBYLL, EPOS-LHC) cross section predictions are also included. This includes the statistical (outer/thinner error bar) and the systematic (inner/thicker error bar).

\section{Proton-Proton Cross section}

From the TA proton-air cross section result we can determine the total proton-proton cross section. The process of inferring $\sigma_{p-p}$ from $\sigma_{p-\text { air }}^{\text {inel }}$ is described in details in [27] and [28]. 


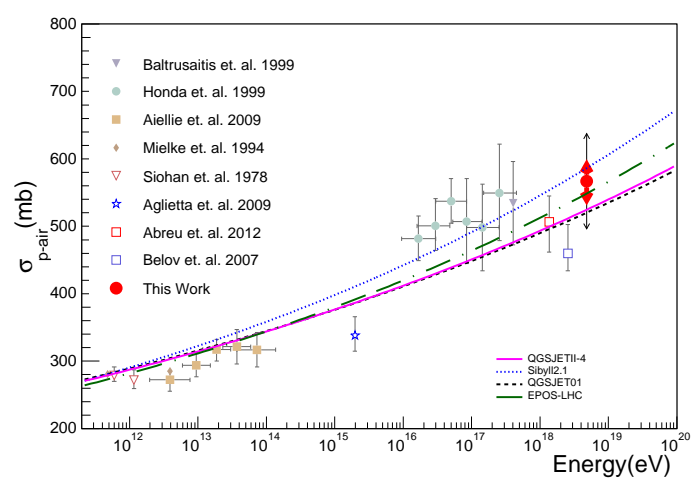

Figure 2: The proton-air cross section result of this work, including the statistical (outer/thinner) and systematic (inner/thicker) error bars. The result of this work is shown in comparison to other experimental results ( [20], [15], [21], [22], [23], [24], [25], [26]). In addition, the high energy models (QGSJETII.4, QGSJET01, SIBYLL, EPOS-LHC) cross section predictions are also shown by solid line, fine dashed line, dotted line, and dashed line consecutively.

The $\sigma_{p-p}$ is calculated from the measured cross section, also known as the inelastic cross section $\sigma_{p-\text { air }}^{\text {inel }}$, using both Glauber Formalism [29].

The relation between the $\sigma_{p-\text { air }}^{\text {inel }}$ and the $\sigma_{\mathrm{p}-\mathrm{p}}$ is highly dependent on the forward scattering elastic slope $B$.

$$
B=\frac{d}{d t}\left[\ln \frac{d \sigma_{\mathrm{p}-\mathrm{p}}^{\mathrm{el}}}{d t}\right]_{t=0}
$$

In this proceeding $\sigma_{p-a i r}^{\mathrm{inel}}$ is converted to $\sigma_{p-p}^{\text {total }}$ using Glauber theory and the BHS QCD inspired fit prediction in the $B$ vs. $\sigma_{\mathrm{p}-\mathrm{p}}^{\text {tot }}$ space ( [2], [3]). The $\sigma_{\mathrm{p}-\mathrm{p}}^{\mathrm{total}}$ is found to be $170_{-44}^{+48}[\text { Stat. }]_{-17}^{+19}[$ Sys.] mb.

The $\sigma_{\mathrm{p}-\mathrm{p}}^{\text {total }}$ calculated in this work is shown in Figures 3 compared to previous results from cosmic ray experiments. The dotted curve is the QCD inspired fit of the total p-p cross section vs. the center of mass energy $\sqrt{s}(\mathrm{GeV})$ [5]. The result is in agreement with the fit.

\section{Conclusion and Outlook}

In this proceeding we used events collected by Telescope Array in hybrid mode to determine the $\sigma_{\mathrm{p} \text {-air }}^{\text {inel }}$ using the $K$-Factor method. The hadronic model dependence of the $K$-Factor method was investigated. The latest updated hadronic interaction models have converged with time on the value of $K$ with an uncertainty of $\sim 3 \%$. This makes the $K$-Factor method a weakly model

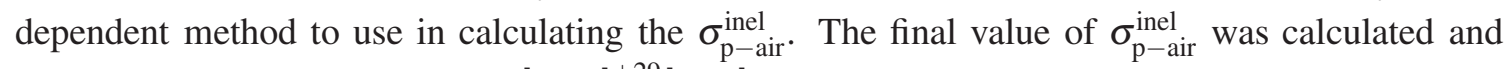
found to be equal to $567.0 \pm 70.5[\text { Stat. }]_{-25}^{+29}[$ Sys. $] \mathrm{mb}$.

Ultimately the value of $\sigma_{\mathrm{p}-\mathrm{p}}$ is determined from $\sigma_{\mathrm{p}-\text { air }}^{\text {inel }}$ using Glauber theory and BHS QCD inspired fit. Such a fundamental measurement at this high energy $(\sqrt{s}=95 \mathrm{TeV})$ could not be obtained with current particle accelerators. The value of $\sigma_{\mathrm{p}-\mathrm{p}}^{\mathrm{tot}}$ was determined to be $170_{-44}^{+48}[\text { Stat. }]_{-17}^{+19}[$ Sys. $]$ $\mathrm{mb}$. 


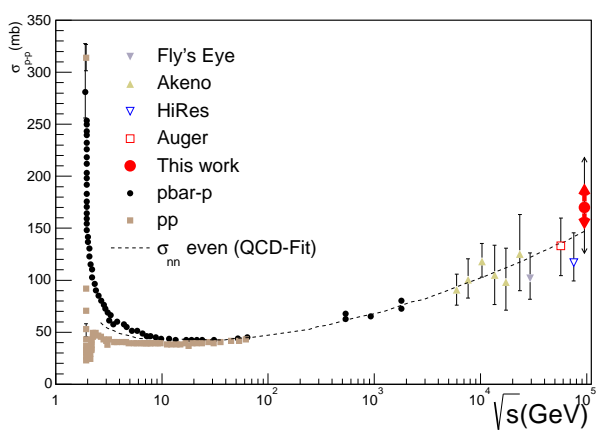

Figure 3: The proton-proton cross section vs. the center of mass energy result of this work, including the statistical (outer/thinner) and systematic (inner/thicker) error bars propagated from the proton-air cross section statistical and systematic errors calculation. The $\bar{p} \mathrm{p}$ and the $\mathrm{pp}$ data are shown in smaller darker circles and square symbols consecutively. The result of this work is shown in comparison to previous work by cosmic rays detectors ( [15], [21], [23], [25]). The dashed curve is the QCD inspired fit [5]. This plot is adapted from [5].

While the events used in this analysis were collected with MD-SD part of the detector, future cross section results, using thoroughly analysed events could be performed using the rest of the detector. This will enable us to study the measurement down to $1 \mathrm{EeV}$ with higher statistical power which would allow us to constrain the available high energy model cross section predictions.

\section{References}

[1] Ralf Ulrich, Ralph Engel, and Michael Unger. Phys.Rev., D83:054026, 2011.

[2] M.M. Block. Ultra-high Energy Predictions of Proton-Air Cross Sections from Accelerator Data: an Update. Phys.Rev., D84:091501, 2011.

[3] M.M. Block, F. Halzen, and T. Stanev. Phys.Rev., D62:077501, 2000.

[4] C. Avila et al. Phys.Lett. B, 445, 1999.

[5] M.M. Block and F. Halzen. Phys.Rev., D72:036006, 2005.

[6] S. Ostapchenko. Nucl.Phys.Proc.Suppl., 151:143-146, 2006.

[7] N.N. Kalmykov, S.S. Ostapchenko, and A.I. Pavlov. Nucl.Phys.Proc.Suppl., 52B:17-28, 1997.

[8] Eun-Joo Ahn, Ralph Engel, Thomas K. Gaisser, Paolo Lipari, and Todor Stanev. Cosmic ray interaction event generator SIBYLL 2.1. Phys.Rev., D80:094003, 2009.

[9] T. Pierog, Iu. Karpenko, J.M. Katzy, E. Yatsenko, and K. Werner. 2013.

[10] Till Bergmann et al. Astropart.Phys., 26:420-432, 2007.

[11] T. Pierog et al. Nucl. Phys. Proc. Suppl., 151:159-162, 2006. 
[12] G. Bossard et al. Phys. Rev., D63:054030, 2001.

[13] D. Heck et al. CORSIKA: A Monte Carlo code to simulate extensive air showers. 1998.

[14] R.U. Abbasi et al. Astropart.Phys., 64:49-62, 2014.

[15] R.M. Baltrusaitis et al. Phys.Rev.Lett., 52:1380-1383, 1984.

[16] C.L. Pryke. Astropart.Phys., 14:319-328, 2001.

[17] T. Abu-Zayyad et al. Phys.Rev., D88(11):112005, 2013.

[18] J. Abraham et al. Astropart.Phys., 31:399-406, 2009.

[19] Mariangela Settimo. In International Cosmic Ray Conference, 2011.

[20] F. Siohan et al. J.Phys., G4:1169-1186, 1978.

[21] M. Honda et al. Phys.Rev.Lett., 70:525-528, 1993.

[22] H.H. Mielke et al. J.Phys., G20:637-649, 1994.

[23] K. Belov. Nucl.Phys.Proc.Suppl., 151:197-204, 2006.

[24] G. Aielli et al. Phys.Rev., D80:092004, 2009.

[25] Pedro Abreu et al. Phys.Rev.Lett., 109:062002, 2012.

[26] M. Aglietta et al. Phys. Rev. D, 79:032004, Feb 2009.

[27] Ralph Engel, T.K. Gaisser, Paolo Lipari, and Todor Stanev. Phys.Rev., D58:014019, 1998.

[28] T.K. Gaisser, U. Sukhatme, and G.B. Yodh. Phys.Rev., D36:1350, 1987.

[29] R.J. Glauber and G. Matthiae. Nucl.Phys., B21:135-157, 1970. 